\title{
Cognitive and Behavioral Profiles of Children with Fetal Alcohol Spectrum Disorders
}

\author{
Piyadasa Kodituwakku • Elizabeth Kodituwakku
}

Published online: 17 June 2014

(C) Springer International Publishing Switzerland 2014

\begin{abstract}
The last two decades have witnessed a rapid growth of published data on the cognitive and behavioral functioning of children with fetal alcohol spectrum disorders (FASD). The main of aim of this paper is to review cognitive and behavioral data on FASD using a causal modeling framework originally proposed by Morton and Frith [1, 2], according to which data pertinent to a neurodevelopmental disorder can be organized at three levels: neurobiology, cognition, and behavior. In this review, we confine ourselves to reviewing the studies of cognitive abilities and behavior in children with FASD. The studies of cognitive functions can be further divided into two groups: those of elementary functions (e.g., reflexive orienting responses) and those of complex functions (e.g., language, memory). There is evidence that children with FASD are slower at reflective orienting responses and exhibit deficits in associative learning. The pattern of results emerging from the studies of complex cognitive functions is that the FASD group shows performance decrements with increased task complexity. At the behavioral level, children with prenatal alcohol exposure are rated as showing deficient adaptive skills, particularly in the social domain. These children have also been observed to show deficits in executive functioning with notable behavioral and emotional regulatory problems. A broad range of risk factors including the alcohol's teratogenicity seem to interactively contribute to these cognitive and behavioral problems.
\end{abstract}

Keywords Fetal alcohol syndrome (FAS) - Alcohol related neurodevelopmental disorder (ARND) · Fetal alcohol spectrum disorder (FASD) · Neurocognitive profile .

Behavioral phenotype

P. Kodituwakku $(\square) \cdot$ E. Kodituwakku

Center for Development and Disability, University of New Mexico

School of Medicine, 2300 Menaul NE, Albuquerque, NM 87107,

USA

e-mail: pkodituwakku@salud.unm.edu

\section{Introduction}

'Fetal alcohol spectrum disorder' (FASD) is a broad term that refers to a range of morphological anomalies and cognitivebehavioral deficits seen in children with prenatal alcohol exposure. At one end of the spectrum are severely affected children who show a characteristic cluster of malformations called fetal alcohol syndrome, which is characterized by a pattern of minor anomalies on the face, growth restrictions and central nervous system dysfunction [3]. It is now known that the majority of children on the spectrum show only some or none of the above morphological anomalies, yet display significant cognitive and behavioral problems. The term, alcohol related neurodevelopmental disorder (ARND), has been introduced to describe such neurodevelopmental problems that are seen in the absence of clinically discernable morphological anomalies [4]. Identification of children with ARND is clinically challenging because the neurocognitive profile of these children substantially overlaps with the profiles seen in other neurodevelopmental disorders, notably in attention deficit hyperactivity disorder (ADHD) and conduct disorder. Therefore, the question of whether children with FASD display a unique neurocognitive profile has received considerable attention in recent years [5].

Since the deleterious effects of prenatal alcohol exposure were first described in the medical literature over 40 years ago $[3,6]$, a large body of data on neurocognitive functioning in alcohol-exposed children has been accumulated. These data come from two main sources: retrospective studies in which clinic-referred or epidemiological samples have been compared with other groups without alcohol exposure, and prospective studies in which large cohorts have been followed up from infancy through adulthood. While most investigators have utilized standardized tests (e.g., IQ tests) or test batteries (e.g., Delis-Kaplan, CANTAB) to assess cognitive functioning and behavior in children with FASD, some have employed 
experimental tests. Since the standardized tests are typically 'broad-band' tests designed to assess complex functions, some investigators have utilized 'narrow-band' tests to probe elementary functions such as eyeblink conditioning and saccades.

To organize the data related to neurobehavioral functioning in FASD, we have used a framework [5, 7] based on the causal modeling approach to neurodevelopmental disorders proposed by Morton and Frith [1, 2]. Within this framework, the outcomes of prenatal alcohol exposure can be organized in a hierarchical order: neurobiological, cognitive and behavioral (see Fig. 1). Since there is a growing body of literature on elementary functions, we have grouped cognitive data under elementary and complex functions. Because other papers in the current volume present reviews of neurobiological findings, we confine ourselves to reviewing the observations on cognition and behavior.

\section{Elementary Functions}

The study of intermediate phenotypes or endophenotypes has proven to be a useful strategy to probe functional deficits associated with neurodevelopmental disorders such as autism and attention deficit hyperactivity disorder. Gottesman and Gould [8] define endophenotypes as "measurable components unseen by the unaided eye along the pathway between distal genotype and disease" (p. 636). Therefore, being closer to the source of pathology, endophenotypes are considered 'biomarkers' of a neurogenetic disorder. A range of elementary functions including sensory motor gating, eye tracking (saccades), P300 event related potential and eyeblink conditioning have been investigated as candidate endophenotyes in a number of neuropsychiatric disorders $[8,9]$. In the area of FASD, researchers have investigated two elementary functions: eyeblink conditioning and reflexive orienting responses [10].
Eyeblink Conditioning (EBC)

$\mathrm{EBC}$ is an associative learning paradigm in which the eyeblink reflex is conditioned to a neutral stimulus by pairing the neutral stimulus (tone) with an unconditioned stimulus (an air puff to the eye) [11]. In the standard delay conditioning paradigm, a tone [conditioned stimulus (CS)] is presented just before an air puff to the eye [unconditioned stimulus (US)] and the two stimuli are terminated at the same time. In trace conditioning, however, a short time interval ( 500 to $1,000 \mathrm{~ms}$ ) is inserted between the offset of the tone and the onset of the air puff. It is now known that the cerebellum plays a critical role in both types of conditioning, but the hippocampus is involved only in trace conditioning. Since the cerebellum and hippocampus are both sensitive to the deleterious effects of alcohol, EBC as a potential biomarker of alcohol-induced brain damage has been investigated in both pre-clinical and clinical studies $[12,13]$.

Evidence from pre-clinical and clinical studies show that animals with prenatal alcohol exposure exhibit deficits in the acquisition of $\mathrm{EBC}$, as indicated by lateonset or late-peaked conditioned responses [12, 14]. Similarly, Jacobson et al. [13] found that children exposed to alcohol prenatally were markedly impaired in EBC. In the Jacobson et al. study, none of the children with FAS reached the conditioning criteria, whereas 75 $\%$ of the controls did, which was a substantial group difference. However, Coffin et al. [15] obtained evidence that children with dyslexia and those with FASD were all impaired at EBC, indicating that deficient EBC was not unique to FASD.

\section{Orienting Responses}

It is well known that one will reflexively orient attention to a salient event in the visual field, a phenomenon known as
Fig. 1 Causal modeling framework of cognitivebehavioral functioning in FASD (adapted from Morton and Frith, 1995)

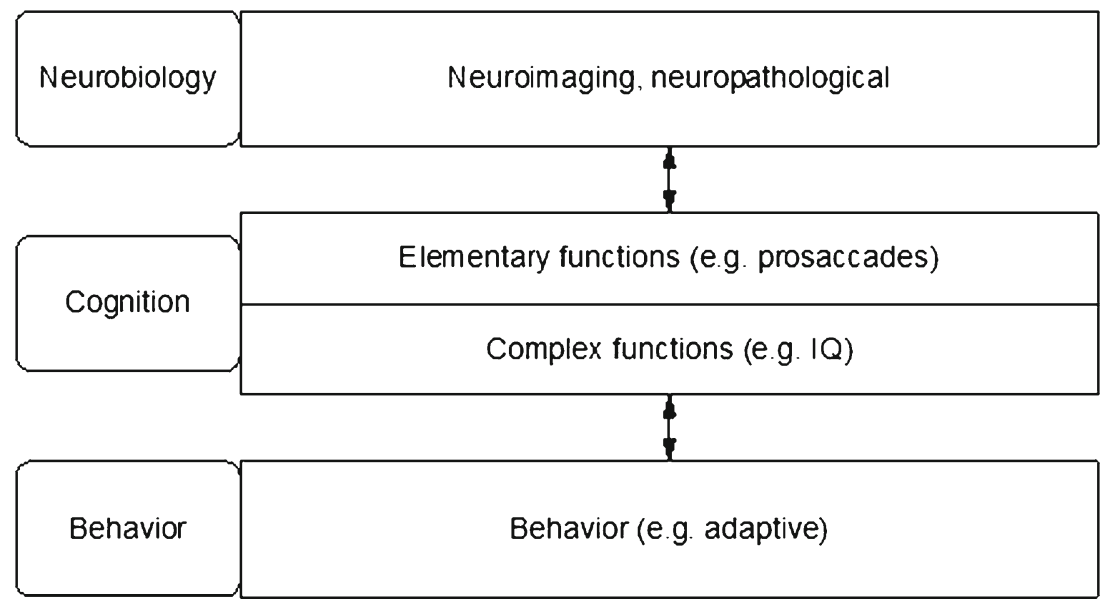


capture of attention [16]. Similarly, an orienting reflex toward a sound is present in the auditory domain. These reflexes have received considerable attention as potential endophenotypes of neurogenetic disorders because they are subserved by welldefined subcortical neural circuits [17] and because they may reveal markers of aberrant early developmental processes.

\section{Auditory Orienting Responses}

It is known that the vagus nerve responds to metabolic changes in the brain by regulating the heart's pacemaker [18]. Therefore, researchers have used heart rate as an index of metabolic changes in the brain in response to sensory stimulation [19]. The association between heart rate and information processing has allowed investigators to examine orienting responses in infants [20]. Kable and Coles [21] utilized this methodology to investigate orienting responses to auditory stimuli by assessing heart rate deceleration in 6-month-old infants with prenatal alcohol exposure. These investigators found that alcohol-exposed infants responded slower to auditory stimuli, but showed higher levels of arousal than neurotypical controls.

Stephen et al. [22] investigated neural correlates of auditory processing in preschoolers with prenatal alcohol exposure using magnetoencephalography (MEG). The investigators recorded MEG while children listened to a series of tones, which comprised frequent $(1000 \mathrm{~Hz}, 72 \mathrm{~dB})$ and infrequent tones $(1200 \mathrm{~Hz} ; 72 \mathrm{~dB})$ presented in a ratio of $84: 16$. The location and time course of neurophysiological responses to these tones were assessed using a multipole spatio-temporal modeling technique. Results showed significant delays in M100 and M200 latencies in responses for the FASD group compared to the control. This finding indicates a slowness of the FASD group even at the early stages of information processing.

\section{Prosaccades}

Anomalies in reflexive eye tracking have been observed in a number of neurodevelopmental disorders [23], including fetal alcohol spectrum disorders $[24,25]$. The standard paradigm used to assess saccades involves having the participant move his or her eye gaze from a central fixation point toward a sudden onset peripheral stimulus (prosaccade), or to look away from the peripheral target toward its mirror image location (antisaccade). Green et al. [24, 25] found that children with FASD showed longer reaction times during prosaccades, suggesting that they had difficulty with the initiation of responses.

Our research team [26] sought to characterize the neural correlates of deficient eye tracking by recording MEG during the performance of a prosaccade task by adolescents with FASD. In line with the findings from the auditory domain [22], the results of this study showed that M100 signals from the FASD group during prosaccades were slower than those from the control group. Therefore, evidence converging from behavioral and neuroimaging studies shows that children and adolescents with FASD are slower at the processing of sensory data.

\section{Complex Functions}

We use the term complex functions to denote those functions that involve effort and that are typically subserved by complex neural circuitries. As shown in Fig. 1, broad neuropsychological test batteries or experimental tasks are utilized to assess these complex abilities. In this section, we will review intellectual functions, attention and executive functions and domain-specific abilities such as language, visual perception, and memory.

\section{Intellectual Functions}

Diminished intellectual functioning (a composite IQ score one to two standard deviations below the mean) is generally considered as a defining characteristic of FASD; as such, it has been used as a criterion of FASD case definitions in surveillance studies and in some diagnostic schemes. In their early studies, Streissguth et al. [27] found that a larger proportion of alcohol exposed individuals had IQs more than two standard deviations below the mean although the range of IQ scores was broad, varying from severe intellectual disability to the average range. These investigators [28] also observed that IQ scores in alcohol-exposed patients remained stable over repeated testing. There is evidence that children with FASD exhibit deficits in both verbal and nonverbal IQs [29], suggesting a generalized pattern of intellectual impairments. Data derived from studies conducted in South Africa [30], Italy [31], Finland [32] and the US [33] all have confirmed a generalized patterns of intellectual deficits. Korkman et al. [32] have obtained evidence that those who were exposed to alcohol throughout pregnancy had greater intellectual deficits than those who were exposed in the first trimester only.

The foregoing studies have mostly included children who have been exposed to substantial amounts of alcohol and who have experienced numerous adverse life conditions, such as poverty and exposure to violence. May et al. [30] found that maternal variables like low education and low socioeconomic status (SES) were associated with lower intellectual ability in children with FASD. This finding raises an important question: do children from middle class background, who are exposed to mild to moderate amounts of alcohol show intellectual deficits? A number of recent studies from Denmark and the UK suggest that mild to moderate prenatal alcohol exposure in children growing up in predominantly in middle class settings do not exhibit intellectual deficits [34-37]. Some 
have suggested that residual confounding factors such as relative affluence may explain why these children don't exhibit IQ deficits associated with moderate levels of alcohol exposure [38, 39]. Data from the Avon Longitudinal Study of Parents and children (ALSPAC) have revealed that abstainers and women drinking 7+ units of alcohol/week came from disadvantaged backgrounds compared to those drinking moderate amounts of alcohol during pregnancy [39]. There is also an emerging body of literature showing that genetic variability in mothers and their offspring may moderate the effects of prenatal alcohol exposure on cognitive functioning [38, 39]. Using the 'Mendelian randomization' [40, 41] approach, Lewis et al. [38] have obtained evidence that four genetic variants in alcohol metabolizing genes were associated with lower IQs in alcohol-exposed children at age 8. Therefore, complex interactions between genetic and environmental factors seem to determine the intellectual ability of children with FASD.

\section{Attention and Executive Functions}

Attention and executive functions have been the focus of numerous studies, because children with FASD have been clinically observed to be impaired in these areas. In one of their early studies, Streissguth et al. [42] found that children with prenatal alcohol exposure were impaired at vigilance, as assessed by a continuous performance task. Prenatal alcohol exposure was found to be associated with increased variability of responses and the number of commission errors. Since then, researchers have sought to delineate the profile of attention in children with FASD using the most fashionable theoretical framework at the time. Using an experimental framework developed by Douglas [43], Nanson and Histcock [44] found that prenatal alcohol exposure was associated with deficient performance on attentional processes such as maintenance of effort and response inhibition. Coles et al. [45] observed impaired performances of the FASD group, compared to ADHD and neurotypical groups, on tests assessing the Encode and Shift components of the experimental paradigm proposed by Mirsky [46]. Kooistra et al. [47] contrasted the performances of FASD, ADHD, and typically developing children on the three main attentional networks formulated by Posner [48]: alerting, orienting, executive (conflict resolution). Results showed that both the FASD and ADHDcombined type groups were significantly slower than the ADHD-inattentive type and typically developing groups in the executive attention network. In a multi-site study, Mattson et al. [33] tested the utility of a broad neuropsychological test battery, including attentional tests, in differentiating children with FASD from ADHD and typically developing groups. Latent profile analyses revealed that relatively demanding tests of executive function (e.g., CANTAB Spatial Working Memory), but not simple attentional tasks (e.g., simple reaction times), differentiated between groups. Additionally, Green et al. [49] found that the FASD group was more impaired than controls on the more complex Choice Reaction Time task of the CANTAB test battery.

The pattern of results emerging from the foregoing studies is that relatively demanding components of attention (e.g., executive attention) are impaired in children with FASD. There now exists a large body of literature on executive control dysfunction in children with FASD (see Rasmussen [50] and Green [49] for reviews). Researchers have obtained evidence that children with FASD are impaired at cognitive planning [31, 49, 51], extra-dimensional set shifting [33, 52, 53], affective set shifting and decision making [52, 54], verbal and design fluency [55, 56], spatial working memory [49], and response inhibition. Assessment of intra-test variability has revealed that children with FASD have greater difficulty with more complex items than simple ones. For example, Aragon et al. [31] found that the FASD group was more impaired than controls at solving complex planning problems, but not simple ones. Similarly, the FASD group has been observed to display greater difficulty with letter fluency than with category fluency [56]; and has greater difficulty with extra-dimensional set shifting than with intra-dimensional set shifting [33].

The foregoing findings on executive function deficits in children with FASD have been obtained by means of standardized or experimental neuropsychological tests. The question of whether these tests predict the alcohol-exposed child's behavior has been raised. Some investigators have reported that performance measures of some tests, particularly those that purport to measure affective set shifting and decision making, predict parent-reported behavioral problems [52]. However, in a recent study, Gross et al. [57] found that parent ratings of behavior and objective measures of executive functioning were highly discrepant. Some investigators have suggested that the laboratory tests and parent and teacher rating scales of executive functioning such as the BRIEF may not measure the same construct. It remains unanswered if ecologically valid tests of executive functioning predict the child's day-to-day behavior better than standard neuropsychological measures. Schonfeld et al. [58] reported that behavioral ratings of executive functioning in children with FASD predicted their social skills.

The question of whether children exposed to mild to moderate amounts of alcohol in utero display deficits in attention and executive functioning has been the focus of a number of recent studies. Underbjerg et al. [59] recently reported the findings from a study of selective and sustained attention in a large cohort of 5-year old, alcohol-exposed children from the Danish National Birth Cohort. These investigators did not find an association between low to moderate levels of alcohol exposure (up to eight drinks per week) and attentional problems in children. However, those who were exposed to more 
than nine drinks per week exhibited diminished attentional skills. The same research group also failed to find a statistically significant association between low to moderate alcohol exposure and behavioral ratings of executive functions on the BRIEF at the 5-year follow-up [60, 61]. These findings suggest that executive dysfunction observed in children with prenatal alcohol exposure may be the product of the interactive effects of multiple factors including the alcohol's teratogenicity, poverty, genetic etc. Recent evidence shows that poverty and low SES are associated with reduced executive control $[62,63]$.

\section{Language}

As we remarked in our previous reviews [5, 7], the studies of language in children with FASD have produced inconsistent results, mainly due to differences in subject characteristics and methodologies. Early prospective studies that included children exposed to low to moderate amounts of alcohol prenatally did not find an association between language development and prenatal alcohol exposure [64-66]. In contrast, early studies of language development in children with substantial amounts of exposure have reported a broad range of impairments, including oral motor, linguistic, and semantic skills $[67,68]$. Carney and Chermak [68] found an age effect, with younger children showing global language delays and older children displaying primarily syntactic deficits. International studies of FASD have provided fresh insights into the nature of language impairments associated with prenatal alcohol exposure. Adnams et al. [69] found that children with fetal alcohol syndrome from an impoverished community in South Africa displayed most pronounced deficits on the language scale of the Griffiths Scale of Cognitive development. Subsequent studies conducted in South African and Italy have revealed impaired skills in grammar comprehension as assessed by the Test for Reception of Grammar [30, 70].

In North America, a number of investigators have sought to characterize the profile of language skills in alcohol-exposed children utilizing standardized test batteries such as the Test of Language Development and the Clinical Evaluation of Language Fundamentals [71, 72]. These investigations reported that alcohol-affected children displayed deficient skills in both expressive and receptive language, which were nonetheless considered commensurate with their reduced intellectual abilities [71]. Wyper et al. [72] found that older children had greater difficulty than typically developing age peers on relatively demanding tasks such as word ordering and morphological comprehension. There is also consistent evidence that older children have difficulty with demanding verbal tasks such as those involving executive functioning (e.g., Letter and Category Fluency) $[55,56]$ and reading subtle social cues (e.g., social communication) $[73,74]$.
As discussed in previous sections, a number of recent reports have indicated that prenatal exposure to low to moderate amounts of alcohol may not be associated with cognitive deficits including language [39]. Zuccolo et al. [39] found that the offspring of women reporting moderate amounts of alcohol before and during early pregnancy had higher scores on Key Stage 2, a test of academic achievement including a measure of vocabulary, than those born to mothers who reported lighter drinking. As mentioned above, those moderate drinkers came from more affluent backgrounds than did lighter drinkers. There is a substantial body of literature showing that language input (e.g., parents' speech) is a robust predictor of the development of syntactic and semantic skills $[75,76]$. Therefore, it is possible that alcohol-exposed children growing up in affluent families benefitted from their enriched environments (e.g., increased language input). O'Leary et al. [77] have, however, underscored the importance of assessing dose and timing of exposure when evaluating the association between prenatal alcohol exposure and language delay. These investigators found that although low levels of alcohol consumption were not associated with language delay, there was a nonsignificant $30 \%$ increase in risk when moderate to heavy exposure took place in the third trimester [78].

\section{Memory and Learning}

The effects of prenatal alcohol exposure on memory and learning have been investigated using standardized neuropsychological test batteries and specific experimental tasks. More recently, investigators have begun to delineate the neural correlates of learning and memory deficits in alcohol exposed children using neuroimaging methods (See Moore et al. in the current issue). The studies that have utilized broad neuropsychological test batteries such as the Wide Range Assessment of Memory and Learning (WRAML) have shown that children with FASD are impaired at both verbal and visual learning [79-81]. Using structurally comparable verbal and visual memory tests (CVLT and Biber Figure Learning), Mattson and Roebuck [82] found that that the FASD group was deficient in learning and recall of information across all measures. However, some investigators who had used the Children's Memory Scale to assess learning and memory had found alcohol exposure related deficits only in the verbal domain (e.g., pair-associate learning) [83, 84].

A number of studies have manipulated the complexity of materials or the level of memory support for the retrieval of the newly learned materials with a view to elucidating the processes underlying memory difficulties in children with FASD. Aragon et al. [31] demonstrated that children with FASD had greater difficulty with learning the locations of nine complex stimuli than nine simple stimuli. These investigators also reported that the FASD group was impaired at free recall of both verbal and nonverbal information, but was 
unimpaired at recognition memory. Other investigators have confirmed the finding that children with FASD are impaired at free recall, but not at recognition memory [85]. Since the prefrontal cortex is known to play a key role in encoding complex information and free recall [86], it is reasonable to hypothesize that a deficit in frontal-hippocampal connectivity may be partly responsible for learning and memory deficits in FASD. Consistent with this hypothesis is the finding that alcohol-exposed children are deficient in source monitoring [87], which is considered to be a frontally mediated process [88]. There is also evidence that the FASD group is impaired on tasks that are known to be sensitive to hippocampal functions, such as the Virtual Morris Water Maze [89] and spatial memory [90].

In recent years, researchers have turned their attention to delineating the neural correlates of memory in children with FASD. Sowell et al. [91] examined functional MRI activation patterns in children with FASD and typically developing controls during the performance of a paired associate learning task. While the typically developing group displayed increased activation in the left medial temporal lobe, left dorsal frontal lobe, and bilateral temporal cortices, the FASD group showed reduced activation in these regions. Differences in functional MRI activation patterns have also been observed during the performance of working memory tasks by children with FASD and typically developing controls $[92,93]$.

\section{Visual Spatial and Visual Constructional}

Surprisingly, perceptual skills in children with FASD have received only scant attention, despite the fact that numerous ocular abnormalities [94, 95] and hearing impairments [96] have been reported in children with prenatal alcohol exposure. Uecker and Nadel [90] reported that children with FASD were unimpaired on a test of facial recognition, but Kaemingck et al. [80] found that the FASD group showed impaired performance on a more demanding angular matching task. Recent magnetoencephalographic studies have revealed that children with FASD were significantly slower in the processing of visual and auditory information $[22,26]$. These data show that alcohol-exposed children are slower than controls in processing sensory information even at very early stages. Such a slowness can hamper the integration of perceptual information at higher levels of processing.

A number of investigators have reported that children with FASD show deficient visual constructional skills as assessed by copying tasks such as the Beery Visual Motor Integration Test $[80,90]$. It appears that children with FASD show performance decrements in visual construction with increased complexity, a pattern similar to that is observed in the perceptual domain.
Number Processing

The observation that children with FASD tend to perform poorer in math than in other academic subjects [97, 98] have prompted investigators to probe the association between prenatal alcohol exposure and number processing. A seminal study by Kopera-frye et al. [99] showed that the FASD group was unimpaired on simple number processing tasks, but was impaired on complex numerical reasoning tasks such as cognitive estimation. A subsequent study by Jacobson et al. [100] showed that children with substantial prenatal alcohol exposure had a specific deficit in magnitude estimate and that this deficit mediated their difficulties in calculation. The same research group has also reported [101] notable differences in fMRI activation patterns between FASD and typically developing children during the performance of a number processing task. While the control group recruited the fronto-parietal network that has previously been shown to be associated with number processing, the alcohol-exposed group recruited a diffusely distributed network. Santhanam et al. [102] also have demonstrated that children with prenatal alcohol exposure exhibited reduced fMRI activation during the performance of an arithmetic task, particularly in the areas known to be associated with number processing including left superior and right inferior parietal regions and medial frontal gyrus.

\section{Social Cognition}

In view of the evidence that alcohol-exposed animals exhibit deficits in social behavior (e.g., reduced play activities) and that alcohol-exposed humans develop a range of secondary disabilities related to social skills (e.g., inappropriate sexual behavior; trouble with the law), the question of whether those with FASD have a core deficit in social-emotional functioning has been raised [103]. Some evidence in support of the core deficit hypothesis comes from infant research in which alcohol-exposed infants have been found to show differences in temperament. Using the still face paradigm, Haley et al. [104] demonstrated that alcohol-exposed, 5-7-months old infants displayed increased stress reactivity. Molteno et al. [105•] have obtained evidence that 13-month-old infants showed deficits in elicited symbolic play, which predicted their cognitive performance at age 5. Molteno et al. [106] also reported recently that prenatal alcohol exposure was related increased infant emotional withdrawal and decreased activity levels.

Several investigators have sought to delineate sociocognitive and emotional regulatory skills in children and adults with prenatal alcohol exposure. Greenbaum et al. [107] compared FASD, ADHD, and typically developing children on a test battery measuring social cognitive and emotion processing abilities, and found that the FASD group 
showed weaker social cognitive and emotional processing abilities than the other two groups. Other investigators have reported deficits in social problem solving [108], moral maturity [109], and affective prosody comprehension [110] associated with prenatal alcohol exposure.

An important question related to social-cognitive difficulties in children with FASD concerns whether these difficulties are secondary to some other primary process, such as deficient executive functioning and intellectual disabilities. A partial answer to this question was provided in a study by Bishop et al. [111], in which children with autism were compared with those with prenatal alcohol exposure on the Autism Diagnostic Observation Scale (ADOS). Results showed that both groups exhibited difficulty with peers and inappropriate behaviors; however, only the ASD group exhibited difficulty in initiating social interaction, sharing of affect and using nonverbal communication. This pattern of behaviors suggests that deficient social behavior in the FASD group may be associated with executive control dysfunction [58].

\section{Motor}

Because there exist recent systematic reviews of the effects of prenatal alcohol exposure on motor function (see Bay and Kesmodel[112]), we herein focus on the studies published since 2011. Numerous studies have reported that children with heavy prenatal alcohol exposure show deficient fine motor skills as measured by the tests such as the grooved Pegboard and Finger Tapping Test. According to parent reports, children with FASD have significant fine motor, but not gross motor delays [113]. The impact of fine motor deficits on alcoholaffected children's functional activities such as writing is unknown. Therefore, Duval-White et al. [114] examined functional handwriting performance in school age children with FASD using the Process Assessment of the Learner- $2^{\text {nd }}$ Edition and Visual Motor Precision subtest of the developmental neuropsychological assessment (NEPSY). Results showed that children with FASD performed below average range on handwriting legibility and speed. However, since the study design did not include a control group, the authors had not controlled for confounding variables. Simmons and colleagues $[115,116]$ have investigated the alcohol-exposed children's ability to produce isometric (constant) and isotonic (graded) force. These investigators found that the FASD group was less accurate and more variable in regulating force compared to typically developing controls.

The question of whether mild to moderate levels of prenatal alcohol exposure produce motor deficits has been addressed in recent studies. Humphriss et al. [117, 118] investigated the association between prenatal alcohol exposure and childhood balance ability in children from the UK Birth Cohort Study. Children with mild to moderate alcohol exposure did not show deficits in dynamic balance (beam walking), static balance with eyes open and static balance with eyes closed. These findings are consistent with those obtained in previous studies of mild to moderate exposure [112].

\section{Behavioral Profile}

Is there a signature behavioral profile or a behavioral phenotype associated with FASD? Researchers have attempted to answer this question by means of parent and teacher-rated questionnaires. Using a number of questionnaires including the Child Behavior Checklist, Behavioral Rating Inventory, and Conners' Rating Scale, Stevens et al. [119] sought to identify behaviors that are commonly associated with FASD. Results showed that children who were diagnosed with FASD were rated by their caregivers and teachers as having more internalizing problems, externalizing problems and attention problems. Graham et al. [120] compared alcohol-exposed children with or without ADHD, non-exposed children with ADHD and neurotypical children on the Sluggish Cognitive Tempo Questionnaire and found that specific items (e.g. forgets details, confused, forgetful, and drowsy) discriminated the alcohol-exposed group from controls.

A fundamental challenge to employing self-rated or caregiver-rated questionnaires to identify a behavioral profile is the difficulty in controlling for rater-bias. Clinicians are well aware of the fact that caregiver ratings are often influenced by the rater's emotional states, beliefs and socio-cultural background. One strategy to overcome this limitation is the application of the coherence rule, according to which the data obtained from different sources must fit together. Another strategy involves determining the predictive validity of the reported behavior; that is, whether it predicts another independent observation. In a community-based study conducted in Italy, the alcohol-exposed group was rated as showing more inattentive behaviors than controls. The inattentive behaviors, but not hyperactive behaviors, were found to be associated with their school problems [121]. Using the coherence rule, one can hypothesize that increased inattentive behaviors are associated with slow information processing on neuropsychological tests. A previous report on the neurobehavioral profile identified 'acting young for age' as a characteristic central to FASD [122]. It can be considered coherent with general cognitive disabilities in alcohol-exposed children.

Other consistent findings from the studies of behavior in heavily exposed children include increased incidence of psychiatric problems (See the paper by O'Connor in the current issue) and diminished adaptive skills. Numerous studies have documented that children with FASD have deficient adaptive skills across different domains of functioning (e.g., communication, daily living, socialization and motor). A key question pertinent to defining a behavioral phenotype concerns if children with FASD display an uneven profile of adaptive skills. A number of reports have now documented that difficulties in 
socialization is more pronounced in alcohol-exposed children, particularly when they grow older [123-126]. There is some evidence to suggest that deficient executive function predicts social deficits in children with FASD [58]. O'Connor et al. [127] have reported that adolescents with FASD respond well to social skills training, suggesting that social skills deficits in FASD are amenable to interventions. Ware et al. [128] also have found specific patterns of association between executive dysfunction and adaptive behavior. Carr et al. [129] reported that adaptive behavior deficits were associated with sensory processing difficulties, but not with the IQ scores of children with FASD. These deficits in adaptive behaviors have been hypothesized to contribute to various secondary disabilities in this population [130].

The data from the prospective cohort studies that are being conducted in the UK, Denmark, and Australia do not show an association between low to moderate alcohol exposure and increased behavioral problems in offspring [131, 132]. Although some have suggested that effects of alcohol exposure may be masked by factors such as residual confounding [77], the results of these studies highlight the significance of contributions from SES, genetic, and epigenetic factors to the genesis of behavioral and cognitive problems in children with prenatal alcohol exposure.

\section{Conclusions}

In this paper, we have reviewed data from the neurocognitive and behavioral studies of FASD within a hierarchical framework. In our previous reviews, we summarized the findings as showing a generalized difficulty in complex information processing (processing of multiple elements or relations) [7]. This hypothesis is based on the observation that children with FASD show performance decrements with increased task complexity across multiple domains, resulting in relatively parallel profiles of scores for easy and complex tasks (simple planning vs. complex planning; letter fluency vs. category fluency; free recall vs. recognition; implicit memory vs. explicit memory; simple facial recognition vs. recognition of subtle emotional expressions, simple math vs. complex math etc.). Neuroimaging studies of complex information processing have shown that the performance of complex tasks requires the recruitment of multiple regions in the brain [133]. We also hypothesized that the slower processing of information associated with reduced white matter and anomalous grey matter underlies complex processing difficulty. The MEG data reported by Stephen et al. and Coffman et al. [22, 26] seem to instantiate this hypothesis. These investigators found that the FASD group was slower even at very early stage of information processing (at $100 \mathrm{~ms}$ ). This pattern of performance at the neurocognitive level is congruent with some of the behaviors frequently observed at the behavioral level (sluggish tempo, acting young for his or her age, poor self-regulation, poor social skills etc.).

Although we are able to discern patterns in the existing data, we acknowledge that there is considerable variability. The discrepancy between the data obtained from children born to high-risk mothers, mostly from ethnic minorities in North America and the data obtained from children born to relatively affluent mothers in Europe or Australia clearly illustrate this point. In the states of the US where a surveillance study was conducted by the Centers for Disease Control and Prevention (CDC), mothers of children with FAS were found to have specific characteristics [134]. Compared to all mothers of those states, mothers who had children with FAS were more likely to be older, Native American, African American not Hispanic, unmarried, unemployed, to smoke during pregnancy, to have lower educational level and to have more live children. After birth, many of these children experience numerous adverse conditions. Therefore, poorer performance of children growing up in such environments is a result of multiple risk factors. Even among these at risk children, there is variability of test performance related to their ethnic backgrounds. Therefore, it may be reasonable to speak of multiple cognitive and behavioral profiles than a single profile. Despite the considerable variability of test data, having a theoretical framework will help organize the data and generate new ideas to move the field forward.

\section{Compliance with Ethics Guidelines}

Conflict of Interest Piyadasa W. Kodituwakku and Elizabeth Kodituwakku declare that they have no conflict of interest.

Human and Animal Rights and Informed Consent This article does not contain any studies with human or animal subjects performed by any of the authors.

\section{References}

Papers of particular interest, published recently, have been highlighted as:

- Of importance

1. Morton J, Frith U. Causal modeling: a structural approach to developmental psychopathology, in Developmental psychopathology. In: Cicchetti D, Cohen DJ, editors. Theory and methods, vol. 1. New Yor: Wiley; 1995. p. 357-90.

2. Morton J. Understanding developmental disorders. Oxford, UK: Blackwell Publishing; 2004.

3. Jones KL et al. Pattern of malformation in offspring of chronic alcoholic mothers. Lancet. 1973;1(7815):1267-71.

4. Stratton, K., C. Howe, and F. Battaglia, eds. Fetal alcohol syndrome: diagnosis, epidemilogy, prevention, and treatment. 1996, National Academy Press: Washington, D.C. 
5. Kodituwakku PW. Defining the behavioral phenotype in children with fetal alcohol spectrum disorders: a review. Neurosci Biobehav Rev. 2007;31(2):192-201.

6. Lemoine $\mathrm{P}$ et al. Les enfants des parents alcooliques: Anomalies observees apropos de 127 cas. Ouest Med. 1968;21:476-82.

7. Kodituwakku PW, Segall JM, Beatty GK. Cognitive and behavioral effects of prenatal alcohol exposure. Future Neurol. 2011;6(2):237-59.

8. Gottesman II, Gould TD. The endophenotype concept in psychiatry: etymology and strategic intentions. Am J Psychiatry. 2003;160(4):636-45.

9. Johannesen JK et al. Diagnostic specificity of neurophysiological endophenotypes in schizophrenia and bipolar disorder. Schizophr Bull. 2013;39(6):1219-29.

10. Kodituwakku PW, Segall JM, Beatty GK. Cognitive and behavioaral effects of prenatal alcohol exposure. Future Neurol. 2011;6(2):237-59.

11. Clark RE, Squire LR. Classical conditioning and brain systems: the role of awareness. Science. 1998;280(5360):77-81.

12. Stanton ME, Goodlett CR. Neonatal ethanol exposure impairs eyeblink conditioning in weanling rats. Alcohol Clin Exp Res. 1998;22(1):270-5.

13. Jacobson SW et al. Impaired eyeblink conditioning in children with fetal alcohol syndrome. Alcohol Clin Exp Res. 2008;32(2): 365-72.

14. Brown KL, Calizo LH, Stanton ME. Dose-dependent deficits in dual interstimulus interval classical eyeblink conditioning tasks following neonatal binge alcohol exposure in rats. Alcohol Clin Exp Res. 2008;32(2):277-93.

15. Coffin $\mathrm{JM}$ et al. Impaired cerebellar learning in children with prenatal alcohol exposure: a comparative study of eyeblink conditioning in children with ADHD and dyslexia. Cortex. 2005;41(3):389-98.

16. Jonides J. Voluntary vs. automatic control over the mind's eye's movement. In: Long JB, Baddeley A, editors. Attention and performance. Hillsdale: Erlbaum; 1981. p. 187-203.

17. Fecteau JH, Bell AH, Munoz DP. Neural correlates of the automatic and goal-driven biases in orienting spatial attention. $\mathrm{J}$ Neurophysiol. 2004;92(3):1728-37.

18. Porges SW. Orienting in a defensive world: mammalian modification of our evolutionary heritage: A polyvagal theory. Psychophysiology. 1998;32:301-18.

19. Graham FK, Clifton RK. Heart-rate change as a component of the orienting response. Psychol Bull. 1966;65(5):305-20.

20. Clifton RK, Meyers WJ. The heart-rate response of fourmonth-old infants to auditory stimuli. J Exp Child Psychol. 1969;7(1):122-35.

21. Kable JA, Cole CD. The impact of prenatal alcohol exposure on neurophysiological encoding of environmental events at six months. Alcohol Clin Exp Res. 2004;28(3): 489-96.

22. Stephen JM et al. Delays in auditory processing identified in preschool children with FASD. Alcohol Clin Exp Res. 2012;36(10):1720-7.

23. Sweeney JA et al. Eye movements in neurodevelopmental disorders. Curr Opin Neurol. 2004;17(1):37-42.

24. Green CR et al. Deficits in eye movement control in children with fetal alcohol spectrum disorders. Alcohol Clin Exp Res. 2007;31(3):500-11.

25. Green $\mathrm{CR}$ et al. Oculomotor control in children with fetal alcohol spectrum disorders assessed using a mobile eye-tracking laboratory. Eur J Neurosci. 2009;29(6):1302-9.

26. Coffman BA et al. Primary visual response (M100) delays in adolescents with FASD as measured with MEG. Hum Brain Mapp. 2013;34(11):2852-62.
27. Streissguth AP, Herman CS, Smith DW. Intelligence, behavior, and dysmorphogenesis in the fetal alcohol syndrome: a report on 20 patients. J Pediatr. 1978;92(3):363-7.

28. Streissguth AP, Herman CS, Smith DW. Stability of intelligence in the fetal alcohol syndrome: a preliminary report. Alcohol Clin Exp Res. 1978;2(2):165-70.

29. Mattson SN et al. Neuropsychological comparison of alcoholexposed children with or without physical features of fetal alcohol syndrome. Neuropsychology. 1998;12(1):146-53.

30. May PA et al. Maternal Factors Predicting Cognitive and Behavioral Characteristics of Children with Fetal Alcohol Spectrum Disorders. J Dev Behav Pediatr. 2013;34(5):314-25.

31. Aragon AS et al. Neuropsychological study of FASD in a sample of American Indian children: processing simple versus complex information. Alcohol Clin Exp Res. 2008;32(12):2136-48.

32. Korkman M, Kettunen S, Autti-Ramo I. Neurocognitive impairment in early adolescence following prenatal alcohol exposure of varying duration. Child Neuropsychol. 2003;9(2):117-28.

33. Mattson $\mathrm{SN}$ et al. Further development of a neurobehavioral profile of fetal alcohol spectrum disorders. Alcohol Clin Exp Res. 2013;37(3):517-28.

34. Kelly $\mathrm{Y}$ et al. Light drinking in pregnancy, a risk for behavioural problems and cognitive deficits at 3 years of age? Int J Epidemiol. 2009;38(1):129-40.

35. Kelly YJ et al. Light drinking during pregnancy: still no increased risk for socioemotional difficulties or cognitive deficits at 5 years of age? J Epidemiol Community. 2012;66(1):41-8.

36. Kesmodel US et al. Does Binge Drinking During Early Pregnancy Increase the Risk of Psychomotor Deficits? Alcohol - Clin Exp Res. 2013;37(7):1204-12.

37. Eriksen HLF et al. The effects of low to moderate prenatal alcohol exposure in early pregnancy on IQ in 5-year-old children. BJOG. 2012;119(10):1191-200.

38. Lewis, S.J., et al., Fetal Alcohol Exposure and IQ at Age 8: Evidence from a Population-Based Birth-Cohort Study. Plos One, 2012. 7(11).

39. Zuccolo L et al. Prenatal alcohol exposure and offspring cognition and school performance. A 'Mendelian randomization' natural experiment. Int J Epidemiol. 2013;42(5):1358-70.

40. Smith GD. Mendelian randomization for strengthening causal inference in observational studies: Application to gene X environment interactions (vol 5, pg 527, 2010). Perspect Psychol Sci. 2011;6(3):314.

41. Smith GD, Ebrahim S. 'Mendelian randomization': can genetic epidemiology contribute to understanding environmental determinants of disease? Int J Epidemiol. 2003;32(1):1-22.

42. Streissguth AP et al. Attention, distraction and reaction time at age 7 years and prenatal alcohol exposure. Neurobehav Toxicol Teratol. 1986;8(6):717-25.

43. Douglas VI. Attentional and cognitive problems. In: Rutter M, editor. Developmental neuropsychiatry. New York: Guilford Press; 1983. p. 280-329.

44. Nanson JL, Hiscock M. Attention deficits in children exposed to alcohol prenatally. Alcohol Clin Exp Res. 1990;14(5):656-61.

45. Coles $\mathrm{CD}$ et al. A comparison of children affected by prenatal alcohol exposure and attention deficit, hyperactivity disorder. Alcohol Clin Exp Res. 1997;21(1):150-61.

46. Mirsky AF. In: Lyon GR, Krasnegor NA, editors. Disorders of attention, in Attention, memory, and executive function. Baltimore: Paul H. Brookes Publishing Co; 1996. p. 71-96.

47. Kooistra L et al. Comparing Attentional Networks in Fetal Alcohol Spectrum Disorder and the Inattentive and Combined Subtypes of Attention Deficit Hyperactivity Disorder. Dev Neuropsychol. 2011;36(5):566-77.

48. Posner MI, Petersen SE. The Attention System of the Human Brain. Annu Rev Neurosci. 1990;13:25-42. 
49. Green $\mathrm{CR}$ et al. Executive function deficits in children with fetal alcohol spectrum disorders (FASD) measured using the Cambridge Neuropsychological Tests Automated Battery (CANTAB). J Child Psychol Psychiatry. 2009;50(6):688-97.

50. Rasmussen C. Executive functioning and working memory in fetal alcohol spectrum disorder. Alcohol Clin Exp Res. 2005;29(8):1359-67.

51. Kodituwakku PW et al. Specific impairments in self-regulation in children exposed to alcohol prenatally. Alcohol Clin Exp Res. 1995;19(6):1558-64.

52. Kodituwakku PW et al. Emotion-related learning in individuals prenatally exposed to alcohol: an investigation of the relation between set shifting, extinction of responses, and behavior. Neuropsychologia. 2001;39(7):699-708.

53. Carmichael Olson $\mathrm{H}$ et al. Neuropsychological deficits in adolescents with fetal alcohol syndrome: clinical findings. Alcohol Clin Exp Res. 1998;22(9):1998-2012.

54. Kully-Martens K et al. Affective Decision-Making on the Iowa Gambling Task in Children and Adolescents with Fetal Alcohol Spectrum Disorders. J Int Neuropsychol Soc. 2013;19(2):137-44.

55. Schonfeld AM et al. Verbal and nonverbal fluency in children with heavy prenatal alcohol exposure. J Stud Alcohol. 2001;62(2):239-46.

56. Kodituwakku PW et al. Letter and category fluency in children with fetal alcohol syndrome from a community in South Africa. J Stud Alcohol. 2006;67(4):502-9.

57. Gross, A.C., et al., Objective measures of executive functioning are highly discrepant with parent-report in fetal alcohol spectrum disorders. Child Neuropsychol, 2014: p. 1-8.

58. Schonfeld AM et al. Executive functioning predicts social skills following prenatal alcohol exposure. Child Neuropsychol. 2006;12(6):439-52.

59. Underbjerg $\mathrm{M}$ et al. The effects of low to moderate alcohol consumption and binge drinking in early pregnancy on selective and sustained attention in 5-year-old children. BJOG. 2012;119(10):1211-21.

60. Skogerbo A et al. The effects of low to moderate alcohol consumption and binge drinking in early pregnancy on executive function in 5-year-old children. BJOG. 2012;119(10):1201-10.

61. Kesmodel US et al. The effect of different alcohol drinking patterns in early to mid pregnancy on the child's intelligence, attention, and executive function. BJOG. 2012;119(10):1180-90.

62. Noble KG et al. Neural correlates of socioeconomic status in the developing human brain. Dev Sci. 2012;15(4):516-27.

63. Noble KG, McCandliss BD, Farah MJ. Socioeconomic gradients predict individual differences in neurocognitive abilities. Dev Sci. 2007;10(4):464-80.

64. Greene T et al. Prenatal alcohol exposure and language development. Alcohol Clin Exp Res. 1990;14(6):937-45.

65. Fried PA, Watkinson B. 36- and 48-month neurobehavioral follow-up of children prenatally exposed to marijuana, cigarettes, and alcohol. J Dev Behav Pediatr. 1990;11(2):49-58.

66. Fried PA, O'Connell CM, Watkinson B. 60- and 72-month followup of children prenatally exposed to marijuana, cigarettes, and alcohol: cognitive and language assessment. J Dev Behav Pediatr. 1992;13(6):383-91.

67. Becker M, Warr-Leeper GA, Leeper Jr HA. Fetal alcohol syndrome: a description of oral motor, articulatory, short-term memory, grammatical, and semantic abilities. J Commun Disord. 1990;23(2):97-124.

68. Carney LJ, Chermak GD. Performance of American Indian children with fetal alcohol syndrome on the test of language development. J Commun Disord. 1991;24(2):123-34.

69. Adnams CM et al. Patterns of cognitive-motor development in children with fetal alcohol syndrome from a community in South Africa. Alcohol Clin Exp Res. 2001;25(4):557-62.
70. Aragon AS et al. Neuropsychological characteristics of Italian children with fetal alcohol spectrum disorders. Alcohol Clin Exp Res. 2008;32(11):1909-19.

71. McGee CL et al. Impaired language performance in young children with heavy prenatal alcohol exposure. Neurotoxicol Teratol. 2009;31(2):71-5.

72. Wyper KR, Rasmussen CR. Language impairments in children with fetal alcohol spectrum disorders. J Popul Ther Clin Pharmacol. 2011;18(2):e364-76.

73. Coggins TE, Timler GR, Olswang LB. A state of double jeopardy: impact of prenatal alcohol exposure and adverse environments on the social communicative abilities of school-age children with fetal alcohol spectrum disorder. Lang Speech Hear Serv Sch. 2007;38(2):117-27.

74. Thorne JC et al. Exploring the utility of narrative analysis in diagnostic decision making: picture-bound reference, elaboration, and fetal alcohol spectrum disorders. J Speech Lang Hear Res. 2007;50(2):459-74.

75. Huttenlocher J. Language input and language growth. Prev Med. 1998;27(2):195-9.

76. Huttenlocher $\mathrm{J}$ et al. Language input and child syntax. Cogn Psychol. 2002;45(3):337-74.

77. O'Leary CM, Bower C. Guidelines for pregnancy: What's an acceptable risk, and how is the evidence (finally) shaping up? Drug and Alcohol Rev. 2012;31(2):170-83.

78. O'Leary $\mathrm{C}$ et al. Prenatal alcohol exposure and language delay in 2-year-old children: the importance of dose and timing on risk. Pediatrics. 2009;123(2):547-54.

79. Kaemingk KL, Mulvaney S, Halverson PT. Learning following prenatal alcohol exposure: performance on verbal and visual multitrial tasks. Arch Clin Neuropsychol. 2003;18(1):33-47.

80. Kaemingk KL, Halverson PT. Spatial memory following prenatal alcohol exposure: more than a material specific memory deficit. Child Neuropsychol. 2000;6(2):115-28.

81. Richardson GA et al. Prenatal alcohol and marijuana exposure: effects on neuropsychological outcomes at 10 years. Neurotoxicol Teratol. 2002;24(3):309-20.

82. Mattson SN, Roebuck TM. Acquisition and retention of verbal and nonverbal information in children with heavy prenatal alcohol exposure. Alcohol Clin Exp Res. 2002;26(6):875-82.

83. Pei JR et al. Memory patterns of acquisition and retention of verbal and nonverbal information in children with fetal alcohol spectrum disorders. Can J Clin Pharmacol. 2008;15(1):e44-56.

84. Willford JA et al. Verbal and visuospatial learning and memory function in children with moderate prenatal alcohol exposure. Alcohol Clin Exp Res. 2004;28(3):497-507.

85. Mattson SN, Riley EP. Implicit and explicit memory functioning in children with heavy prenatal alcohol exposure. J Int Neuropsychol Soc. 1999;5(5):462-71.

86. Balbo J, Shimamura AP. In: Baddeley A, Wilson A, Kopelman M, editors. Frontal lobes and memory, in Handbook of memory disorders. London: John Wiley \& Co; 2002. p. 363-79.

87. Kully-Martens $\mathrm{K}$ et al. Source monitoring in children with and without fetal alcohol spectrum disorders. J Pediatr Psychol. 2012;37(7):725-35.

88. Janowsky JS, Shimamura AP, Squire LR. Source memory impairment in patients with frontal lobe lesions. Neuropsychologia. 1989;27(8):1043-56.

89. Hamilton DA et al. Children with Fetal Alcohol Syndrome are impaired at place learning but not cued-navigation in a virtual Morris water task. Behav Brain Res. 2003;143(1): 85-94.

90. Uecker A, Nadel L. Spatial locations gone awry: object and spatial memory deficits in children with fetal alcohol syndrome. Neuropsychologia. 1996;34(3):209-23. 
91. Sowell ER et al. Functional magnetic resonance imaging of verbal learning in children with heavy prenatal alcohol exposure. Neuroreport. 2007;18(7):635-9.

92. Malisza KL et al. Evaluation of spatial working memory function in children and adults with fetal alcohol spectrum disorders: a functional magnetic resonance imaging study. Pediatr Res. 2005;58(6):1150-7.

93. Norman AL et al. A functional magnetic resonance imaging study of spatial working memory in children with prenatal alcohol exposure: contribution of familial history of alcohol use disorders. Alcohol Clin Exp Res. 2013;37(1):132-40.

94. Stromland K. Visual impairment and ocular abnormalities in children with fetal alcohol syndrome. Addict Biol. 2004;9(2): 153-7. discussion 159-60.

95. Stromland K, Pinazo-Duran MD. Ophthalmic involvement in the fetal alcohol syndrome: clinical and animal model studies. Alcohol Alcohol. 2002;37(1):2-8.

96. Church MW, Kaltenbach JA. Hearing, speech, language, and vestibular disorders in the fetal alcohol syndrome: a literature review. Alcohol Clin Exp Res. 1997;21(3):495-512.

97. Streissguth AP et al. Drinking during pregnancy decreases word attack and arithmetic scores on standardized tests: adolescent data from a population-based prospective study. Alcohol Clin Exp Res. 1994;18(2):248-54.

98. Coles CD, Kable JA, Taddeo E. Math performance and behavior problems in children affected by prenatal alcohol exposure: intervention and follow-up. J Dev Behav Pediatr. 2009;30(1):7-15.

99. Kopera-Frye K, Dehaene S, Streissguth AP. Impairments of number processing induced by prenatal alcohol exposure. Neuropsychologia. 1996;34(12):1187-96.

100. Jacobson JL et al. Number processing in adolescents with prenatal alcohol exposure and ADHD: differences in the neurobehavioral phenotype. Alcohol Clin Exp Res. 2011;35(3):431-42.

101. Meintjes EM et al. An FMRI study of number processing in children with fetal alcohol syndrome. Alcohol Clin Exp Res. 2010;34(8):1450-64.

102. Santhanam $P$ et al. Effects of prenatal alcohol exposure on brain activation during an arithmetic task: an fMRI study. Alcohol Clin Exp Res. 2009;33(11):1901-8.

103. Kelly SJ, Day N, Streissguth AP. Effects of prenatal alcohol exposure on social behavior in humans and other species. Neurotoxicol Teratol. 2000;22(2):143-9.

104. Haley DW, Handmaker NS, Lowe J. Infant stress reactivity and prenatal alcohol exposure. Alcohol Clin Exp Res. 2006;30(12): 2055-64.

105. Molteno CD et al. Infant Symbolic Play as an Early Indicator of Fetal Alcohol-Related Deficit. Infancy. 2010;15(6):586-607. Presents data related to diminished symbolic play skills in young children with FASD.

106. Molteno $\mathrm{CD}$ et al. Infant emotional withdrawal: a precursor of affective and cognitive disturbance in fetal alcohol spectrum disorders. Alcohol Clin Exp Res. 2014;38(2):479-88.

107. Greenbaum RL et al. Social cognitive and emotion processing abilities of children with fetal alcohol spectrum disorders: a comparison with attention deficit hyperactivity disorder. Alcohol Clin Exp Res. 2009;33(10):1656-70.

108. McGee CL et al. Deficits in social problem solving in adolescents with prenatal exposure to alcohol. Am J Drug Alcohol Abuse. 2008;34(4):423-31.

109. Schonfeld AM, Mattson SN, Riley EP. Moral maturity and delinquency after prenatal alcohol exposure. J Stud Alcohol. 2005;66(4):545-54.

110. Monnot $\mathrm{M}$ et al. Neurological basis of deficits in affective prosody comprehension among alcoholics and fetal alcoholexposed adults. J Neuropsychiatry Clin Neurosci. 2002;14(3):321-8
111. Bishop S, Gahagan S, Lord C. Re-examining the core features of autism: a comparison of autism spectrum disorder and fetal alcohol spectrum disorder. J Child Psychol Psychiatry. 2007;48(11):1111-21.

112. Bay B, Kesmodel US. Prenatal alcohol exposure - a systematic review of the effects on child motor function. Acta Obstet Gynecol Scand. 2011;90(3):210-26.

113. Kalberg WO, Buckley D. FASD: what types of intervention and rehabilitation are useful? Neurosci Biobehav Rev. 2007;31(2): 278-85.

114. Duval-White CJ et al. Functional handwriting performance in school-age children with fetal alcohol spectrum disorders. Am J Occup Ther. 2013;67(5):534-42.

115. Simmons RW et al. Children with heavy prenatal alcohol exposure exhibit deficits when regulating isometric force. Alcohol Clin Exp Res. 2012;36(2):302-9.

116. Nguyen TT et al. Children with heavy prenatal alcohol exposure experience reduced control of isotonic force. Alcohol Clin Exp Res. 2013;37(2):315-24.

117. Humphriss $\mathrm{R}$ et al. Balance ability of 7 and 10 year old children in the population: results from a large UK birth cohort study. Int J Pediatr Otorhinolaryngol. 2011;75(1):106-13.

118. Humphriss, R., et al., Prenatal alcohol exposure and childhood balance ability: findings from a UK birth cohort study. BMJ Open, 2013. 3(6).

119. Stevens SA et al. Towards identifying a characteristic neuropsychological profile for fetal alcohol spectrum disorders. 2. Specific caregiver-and teacher-rating. J Popul Ther Clin Pharmacol. 2013;20(1):e53-62.

120. Graham DM et al. Prenatal alcohol exposure, attention-deficit/ hyperactivity disorder, and sluggish cognitive tempo. Alcohol Clin Exp Res. 2013;37 Suppl 1:E338-46.

121. Kodituwakku $P$ et al. Neurobehavioral characteristics of children with fetal alcohol spectrum disorders in communities from Italy: Preliminary results. Alcohol Clin Exp Res. 2006;30(9):1551-61.

122. Nash $\mathrm{K}$ et al. Identifying the behavioural phenotype in Fetal Alcohol Spectrum Disorder: sensitivity, specificity and screening potential. Arch Womens Ment Health. 2006;9(4):181-6.

123. Whaley SE et al. Comparison of the adaptive functioning of children prenatally exposed to alcohol to a nonexposed clinical sample. Alcohol Clin Exp Res. 2001;25(7):1018-24.

124. Thomas SE et al. Comparison of social abilities of children with fetal alcohol syndrome to those of children with similar IQ scores and normal controls. Alcohol Clin Exp Res. 1998;22(2):528-33.

125. Fagerlund A et al. Adaptive behaviour in children and adolescents with foetal alcohol spectrum disorders: a comparison with specific learning disability and typical development. Eur Child Adolesc Psychiatry. 2012;21(4):221-31.

126. Kully-Martens $\mathrm{K}$ et al. A review of social skills deficits in individuals with fetal alcohol spectrum disorders and prenatal alcohol exposure: profiles, mechanisms, and interventions. Alcohol Clin Exp Res. 2012;36(4):568-76.

127. O'Connor MJ et al. A controlled social skills training for children with fetal alcohol spectrum disorders. J Consult Clin Psychol. 2006;74(4):639-48.

128. Ware $\mathrm{AL}$ et al. Executive function predicts adaptive behavior in children with histories of heavy prenatal alcohol exposure and attention-deficit/hyperactivity disorder. Alcohol Clin Exp Res. 2012;36(8):1431-41.

129. Carr JL, Agnihotri S, Keightley M. Sensory processing and adaptive behavior deficits of children across the fetal alcohol spectrum disorder continuum. Alcohol Clin Exp Res. 2010;34(6):1022-32.

130. Streissguth AP et al. Risk factors for adverse life outcomes in fetal alcohol syndrome and fetal alcohol effects. J Dev Behav Pediatr. 2004;25(4):228-38. 
131. Robinson $\mathrm{M}$ et al. Low-moderate prenatal alcohol exposure and risk to child behavioural development: a prospective cohort study. BJOG. 2010;117(9):1139-50.

132. Kelly, Y.J., et al., Light drinking during pregnancy: still no increased risk for socioemotional difficulties or cognitive deficits at 5 years of age? J Epidemiol Community Health, in press.
133. Prabhakaran V et al. Neural substrates of fluid reasoning: an fMRI study of neocortical activation during performance of the Raven's Progressive Matrices Test. Cogn Psychol. 1997;33(1):43-63.

134. Cannon MJ et al. Characteristics and behaviors of mothers who have a child with fetal alcohol syndrome. Neurotoxicol Teratol. 2012;34(1):90-5. 